\title{
Achieving Target Emulsion Drop Size Distributions using Population Balance Equation Models of High Pressure Homogenization
}

\author{
Shashank N. Maindarkar ${ }^{\mathrm{a}}$, Hans Hoogland ${ }^{\mathrm{b}}$, and Michael A. Henson ${ }^{\mathrm{a}, *}$ \\ ${ }^{a}$ Department of Chemical Engineering, University of Massachusetts, Amherst, MA 01003-9303 \\ ${ }^{b}$ Unilever RGD, 3133 AT Vlaardingen, Netherlands
}

*Phone: 413-545-3481; Fax: 413-545-1647; E-mail: henson@ecs.umass.edu 


\section{Supporting Information}

Table S1: Bulk properties of the emulsion system.

\begin{tabular}{|l|l|}
\hline Density of water $\left(\rho_{c}\right)$ & $997.05 \mathrm{~kg} / \mathrm{m}^{3}$ \\
\hline Density of sunflower oil $\left(\rho_{d}\right)$ & $917.36 \mathrm{~kg} / \mathrm{m}^{3}$ \\
\hline Viscosity of water $\left(\eta_{w}\right)$ & $0.88 \mathrm{mPa} . \mathrm{s}$ \\
\hline Viscosity of sunflower oil $\left(\eta_{d}\right)$ & $47.8 \mathrm{mPa} . \mathrm{s}$ \\
\hline Interfacial tension of sunflower oil in water at $25^{\circ} \mathrm{C}$ & $27 \mathrm{mN} / \mathrm{m}$ \\
\hline
\end{tabular}

Table S2: Objective function values at six different homogenization pressures

\begin{tabular}{|c|c|c|}
\hline $\begin{array}{c}\text { Pressure } \\
\text { (bar) }\end{array}$ & $\begin{array}{c}\text { Objective function }(\Psi) \\
\text { using parameters } K_{1}-K_{5} \\
\text { optimized at } 400 \text { bar }\end{array}$ & $\begin{array}{c}\text { Objective function }(\Psi) \\
\text { using parameters } K_{2}-K_{5} \text { optimized } \\
\text { at bar and fitted equations for } K_{1} \text { and } \epsilon\end{array}$ \\
\hline 100 & 0.426 & 0.098 \\
\hline 200 & 0.123 & 0.058 \\
\hline 300 & 0.074 & 0.051 \\
\hline 400 & 0.029 & 0.031 \\
\hline 600 & 0.064 & 0.032 \\
\hline 800 & 0.186 & 0.063 \\
\hline Total & 0.902 & 0.333 \\
\hline
\end{tabular}

Table S3: Model predicted and experimental results for a target DSD generated from a log-normal distribution with $\mu=0.5 \mu \mathrm{m}$ and $\sigma^{2}=0.5$ using a weighting factor of $W=0.01$.

\begin{tabular}{|c|c|c|c|c|c|c|c|c|}
\hline \multirow{2}{*}{$\begin{array}{c}\text { Total surf- } \\
\text {-actant (wt } \%)\end{array}$} & \multicolumn{5}{|c|}{ Pressure (bar) } & \multirow[t]{2}{*}{$\Psi_{2}$} & \multirow[t]{2}{*}{$\Psi_{3}$} & \multirow{2}{*}{$\Psi_{2}+W \Psi_{3}$} \\
\hline & $1^{s t}$ pass & $2^{\text {nd }}$ pass & $3^{r d}$ pass & $4^{\text {th }}$ pass & $5^{\text {th }}$ pass & & & \\
\hline & & & & & & \multicolumn{3}{|c|}{ Model prediction } \\
\hline 1.527 & 736 & - & - & - & - & 0.715 & 4.060 & 0.756 \\
\hline 1.591 & 776 & 617 & - & - & - & 0.177 & 4.590 & 0.223 \\
\hline 1.322 & 800 & 626 & 599 & - & - & 0.027 & 2.703 & 0.054 \\
\hline 1.186 & 766 & 555 & 555 & 566 & - & 0.0060 & 1.883 & 0.025 \\
\hline 1.160 & 234 & 353 & 439 & 487 & 552 & 0.0073 & 1.742 & 0.025 \\
\hline & & & & & & \multicolumn{3}{|c|}{ Experimental result } \\
\hline 1.186 & 766 & 555 & 555 & 566 & - & 0.126 & 1.883 & 0.145 \\
\hline
\end{tabular}


Table S4: Effect of the objective function weighing factor $W$ on model predicted results for four passes and a target DSD generated from a log-normal distribution with $\mu=1.0 \mu \mathrm{m}$ and $\sigma^{2}=0.5$.

\begin{tabular}{|c|c|c|c|c|c|c|c|c|}
\hline $\mathrm{W}$ & $\begin{array}{c}\text { Total surf- } \\
\text {-actant (wt\%) }\end{array}$ & $1^{\text {st }}$ pass & $2^{\text {nd }}$ pass & $3^{\text {rd }}$ pass & $4^{\text {th }}$ pass & $\Psi_{2}$ & $\Psi_{3}$ & $\Psi_{2}+W \Psi_{3}$ \\
\hline 0.001 & 1.345 & 425 & 529 & 546 & 552 & 0.0025 & 2.700 & 0.0052 \\
\hline 0.005 & 1.234 & 600 & 549 & 558 & 565 & 0.0045 & 2.040 & 0.0147 \\
\hline 0.01 & 1.186 & 766 & 555 & 555 & 566 & 0.0060 & 1.880 & 0.0248 \\
\hline 0.05 & 1.043 & 800 & 554 & 530 & 572 & 0.0291 & 1.082 & 0.0832 \\
\hline 0.1 & 0.955 & 532 & 535 & 493 & 571 & 0.0566 & 0.755 & 0.132 \\
\hline
\end{tabular}

